\title{
One Approach for Identification of Brain Signals for Smart Devices Control
}

\author{
Georgi P. Dimitrov$^{1}$, Galina S. Panayotova ${ }^{1}$, Eugenia Kovatcheva ${ }^{1}$, Daniela Borissova ${ }^{2}$ and Pavel \\ Petrov $^{3}$ \\ ${ }^{1}$ University of Library Studies and Information Technologies, Sofia, 1712, Bulgaria \\ 2Institute of Information and Communication Technologies at Bulgarian Academy of Sciences,Acad. G. Bonchev \\ St., Bl. 2, 1113 Sofia, Bulgaria \\ ${ }^{3}$ University of Economics - Varna, Knyaz Boris I Blvd. 77, 9002 Varna, Bulgaria
}

* Corresponding author. Tel.: 0882415054; email: geo.p.dimitrov@gmail.com

Revised submitted June 19, 2018; accepted July 30, 2018.

doi: 10.17706/jsw.13.7.407-413

Abstract: Nowadays the UX design become on a next level. Together with new way of interaction are introduced as finger and hand movement. The technology offer and thought-driven approach with so called brain-computer interface (BCI). This possibility opens new challenges for uses as well as for designers and researchers.

More than 15 years there are devices for brain signal interception, such as EMotiv Epoc, Neurosky headset and others. The reliable translation of user commands to the app on a global scale, with no leaps in advancement for its lifetime, is a challenge. It is still un-solve for modern scientists and software developers. Success requires the effective interaction of many adaptive controllers: the user's brain, which produces brain activity that encodes intent; the BCI system, which translates that activity into the digital signals; the accuracy of aforementioned system, computer algorithms to translate the brain signals to commands. In order to find out this complex and monumental task, many teams are exploring a variety of signal analysis techniques to improve the adaptation of the BCI system to the user. Rarely there are publications, in which are described the used methods, steps and algorithms for discerning varying commands, words, signals and etc.

This article describes one approach to the retrieval, analysis and processing of the received signals. These data are the result of researching the capabilities of Arduino robot management through the brain signals received by BCI.

Key words: Integrated information systems, Brain Computer Interface, BCI, BIG Data, data processing, sensors, EMotiv

\section{Introduction}

The main goal of this article is to highlight the ability to reliably discern the varying brain signals. In a perspective, the clear signal identification will ensure development mathematical tools and algorithms for electrical devices, such as wheelchairs and other similar devices needed by people with reduced communication and motor abilities[1,2]. Also, research will enhance the utilization of the Internet of Things (IoT). Unfortunately, the standard distributed with widespread BCI devices' interface has a very limited command dictionary $[3,4]$. Our team analyses the necessary BCI signals for driving a remote control car. For the needs of our research, we have determined eight simplified base commands - START, STOP, LEFT, RIGHT, FORWARD, BACKWARD, FASTER and SLOWER. In this article the analyses of only two commands - START and STOP is described as well as the differences between these two commands.

The brain emits ElectroEncephaloGraphic(EEG) signals, which are caught by the 5 electrodes of EMOTIV Insight $5+$ device. The raw EEG data is picked up by the electrode and are sent via the Bluetooth into the 
computer. This device can discern 5 signals( Fig. 1):

AF3 - Attention

T7 - Verbal memory

Q1 - Visual Processing

T8 - Emotional memory

AF4 - Judgment
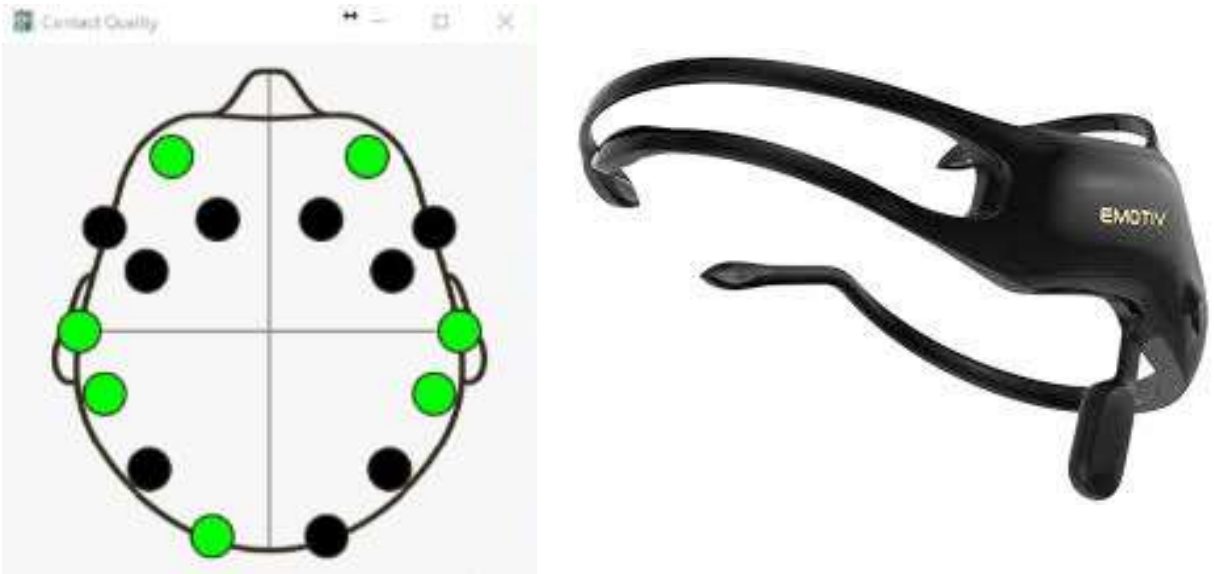

Fig. 1. Map of the electrodes in Epoc Insight 5 chanels.

The received signals are store into database The application BrainData (Fig. 2) developed in university laboratory maintain this process. The application features includes the possibilities for the simultaneous recording and processing of BIG DATA[3]. This means different types of data are recorded at the same time, such as: raw data from $\mathrm{BCI}$, the subject shown at that moment, the video recording of the experiment and other. The system allows interoperability - receiving the data from the BCI system, processing, exporting of the data to other systems and communication with other apps, which can use these data.

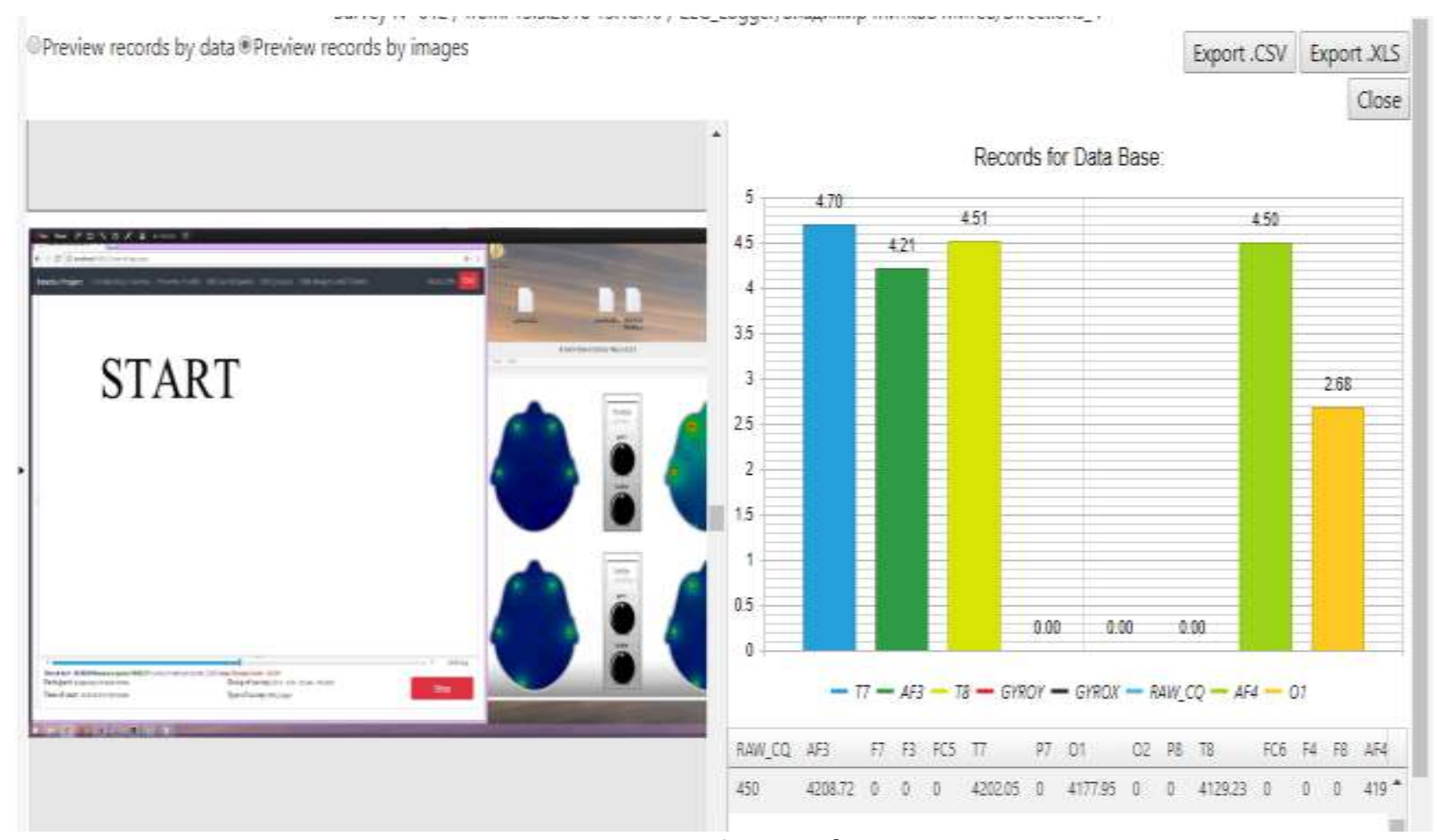

Fig. 2. Brain data.

The steps of creation process are: 1) Obtaining an EEG signal; 2) Inserting data into a database; 3) Filtering 
and classification of EEG signals; 4) Processing of the data array; 5) Utilization of the already processed Smart Device Management data. The focus here is on the step 3 only.

\section{Methods}

\subsection{Data Collection}

The used data are collected during BCI experiments and analyzed offline. It will be described later.

There are 3 examinees. During the experiment, each participant see 8 different pictures (commands). Pictures are changed at different time intervals $-0.25,0.5,1$ and 1.25 seconds in a random principle. Each participant, after seeing the picture, mentally commands and executes it. As was mention above the two opposit commands START and STOP are observed. Each examinee made 7 attempts. Each command is shown more than 360 times in total, or 120 times for each participant.

In real time the signals received from the Epoc are transferred via Bluetooth into the computer. Each trial are recorded 129 rows of data into the database. The count of the records is shown on Table 1. The data are obtained with SDK tool, provided by Emotiv ( github.com/Emotiv/ community-sdk). The collecting information for the experiment, the commands, raw data and screen capture are recorded at the same time into the database, then data are exported and processed using MathLab environment.

Table 1. Count of the Experiments

\begin{tabular}{|l|c|c|}
\hline & \multicolumn{2}{|c|}{ COMMAND } \\
\hline & START & STOP \\
\hline Count of the change pictures & 369 & 367 \\
\hline Count of the rows whit signal data in one experiment & \multicolumn{2}{|c|}{129} \\
\hline All count of records & 100396 & 99674 \\
\hline
\end{tabular}

\subsection{Data Processing}

This research gives a useful insight understanding of the meaning for each Emotiv EEG Sensor. The raw data, received during the display of the "Stop" command are shown on Table 2.

Table 2 Raw data - "START"

\begin{tabular}{|c|c|c|c|c|}
\hline AF3 & T7 & 01 & T8 & AF4 \\
\hline 4233.07 & 4572.24 & 4195.39 & 4188.37 & 4172.35 \\
\hline 4233.52 & 4570.72 & 4225.62 & 4216.47 & 4179.49 \\
\hline$\ldots \ldots \ldots .$. & $\ldots \ldots \ldots$. & $\ldots \ldots \ldots$ & $\ldots \ldots \ldots$. & $\ldots \ldots \ldots$ \\
\hline 4229.48 & 4494.43 & 4231.28 & 4165.12 & 4176.22 \\
\hline
\end{tabular}

The raw data, received during the display of the "Stop" command are shown on Table 3.

Table 3 Raw data - "STOP"

\begin{tabular}{|l|l|l|l|l|}
\hline AF3 & T7 & O1 & T8 & AF4 \\
\hline 4222.33 & 4555.69 & 4158.56 & 4197.81 & 4153.63 \\
\hline 4253.96 & 4683.83 & 4149.65 & 4255.87 & 4136.59 \\
\hline$\ldots \ldots \ldots \ldots \ldots$ & $\ldots \ldots \ldots \ldots . .$. & $\ldots \ldots \ldots \ldots . .$. & $\ldots \ldots \ldots \ldots$ & $\ldots \ldots \ldots \ldots$ \\
\hline 4209.40 & 4500.95 & 4244.27 & 4167.63 & 4147.19 \\
\hline
\end{tabular}

\subsection{Filtering the Signal}

A main characteristics of the data, which are used for research and analysis, are to be accurate and valid. It is rarely possible because when data are copied from reality via a sensor/observer, through transmission and finally stored, it is susceptible to corruption for many reasons[6,7]. During the experiments it is impossible for the test subject to be permanently concentrated upon the given command. This leads to receiving unnecessary or false data in the stream. The original raw data is showing on Figure 3. At first glance the signals have big amplitudes. This data must be filtered.

The filtered stream of data are with exclusion of those whish values differ by $50 \%$ from the average (Figure 4). 


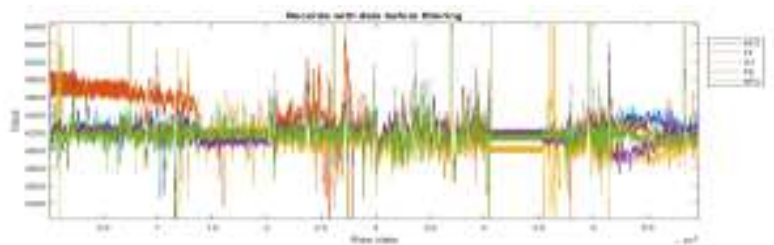

Figure 3 Raw data before filtering

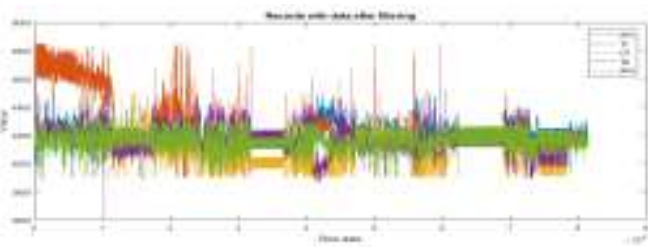

Figure 4 Raw data after filtering

On the Table 4 is shown the count of the records before and after filtering and percent of the excluded rows.

Table 4 rows and filtered rows

\begin{tabular}{|l|c|c|}
\hline & START & STOP \\
\hline All rows with data & 100396 & 96794 \\
\hline Filtered rows & 81432 & 81395 \\
\hline$\%$ excluded records & $19 \%$ & $16 \%$ \\
\hline
\end{tabular}

\subsection{Processing Data}

To distinguish data for different command is a crucial for this research, i.e. the identification of the data[8]. This is arrange by extracting the mean values, standard deviation and differentiate the meaning of the signal values on the each channel. The availability of distinct differences would indicate the possibility of distinguishing the incoming signals.

Mean value of the signal

The avg. values of each channel is calculated in a Mathlab environment:

The resulting avg. values are shown on Table 5.

Table 5. AVG Values for Each Command and Each of the Channels

\begin{tabular}{|l|l|l|l|}
\hline & & \multicolumn{2}{|c|}{ AVA. values } \\
\hline & & START & STOP \\
\hline AF3 & & 4200,83764287520 & 4206,90253712517 \\
\hline T7 & & 4261,67373675012 & 4259,72984825013 \\
\hline 01 & 4103,32124187518 & 4104,19762087510 \\
\hline T8 & & 4166,84227274986 & 4165,75719124990 \\
\hline AF4 & 4156,81051724984 & 4159,94656387485 \\
\hline
\end{tabular}

The graph for the resulting values is shown in the Fig. 5.

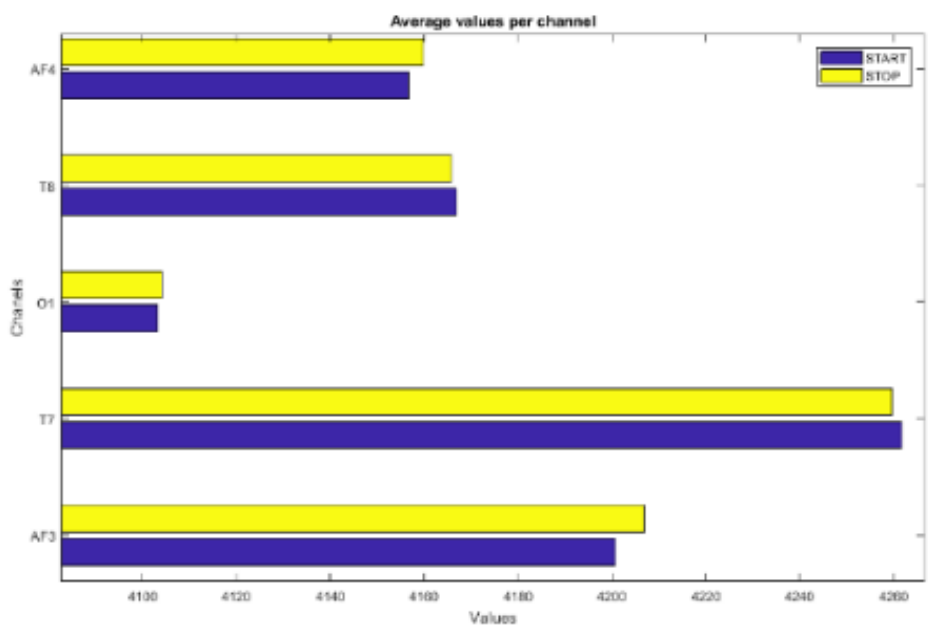

Fig. 5. AVG values for each command and each of the channels.

\section{Standard deviation}

The next step is calculating the standard deviation for each command and each of the channels.

The results show the following: The deviation is biggest at channel T7 and lowest at channels AF3 and AF4. In other words, AF3 and AF4 have the most durable signals, as shown in Table 6 and Fig. 6. 
Table 6. STD Values for each Command and Each of the Channels

\begin{tabular}{|l|c|c|}
\hline Chanel & STDEV(,START”) & STDEV(,STOP”) \\
\hline AF3 & 79.4551 & 78.2322 \\
\hline T7 & 187.8361 & 184.6027 \\
\hline 01 & 100.1024 & 98.2479 \\
\hline T8 & 85.1357 & 86.1827 \\
\hline AF4 & 63.4918 & 62.3431 \\
\hline
\end{tabular}

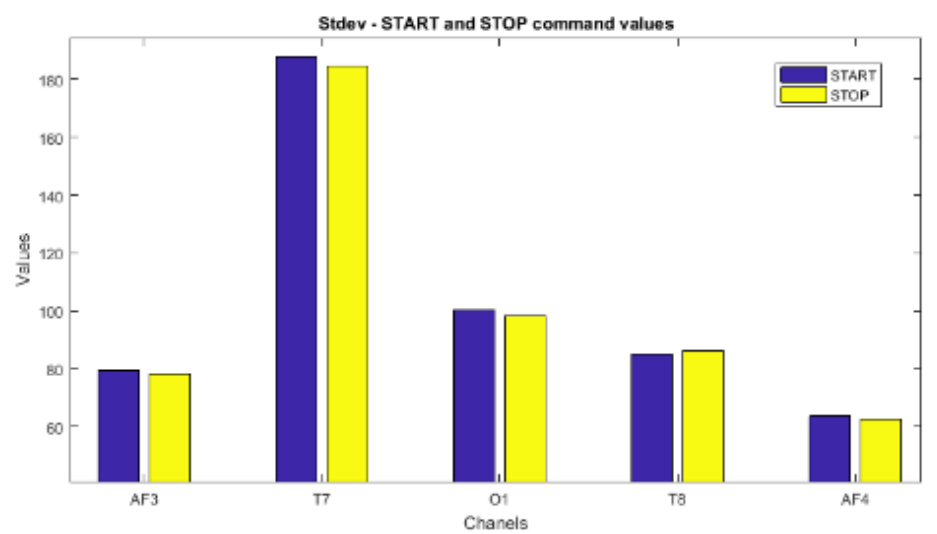

Fig. 6. Graph with STD values for each command and each of the channels.

The differences between the mean values in every channel

In a Mathlab environment we calculated the differences between the mean values of the signals for each channel separately.

The resulting differences are shown in Table 7.

Table 7. Differences between the Values for Each Channel

\begin{tabular}{|l|c|}
\hline & START - STOP \\
\hline AF3 & -6.0649 \\
\hline T7 & 1.9439 \\
\hline 01 & -0.8764 \\
\hline T8 & 1.0851 \\
\hline AF4 & -3.1360 \\
\hline
\end{tabular}

Down are shown the differences between the values of every command in each individual channel.
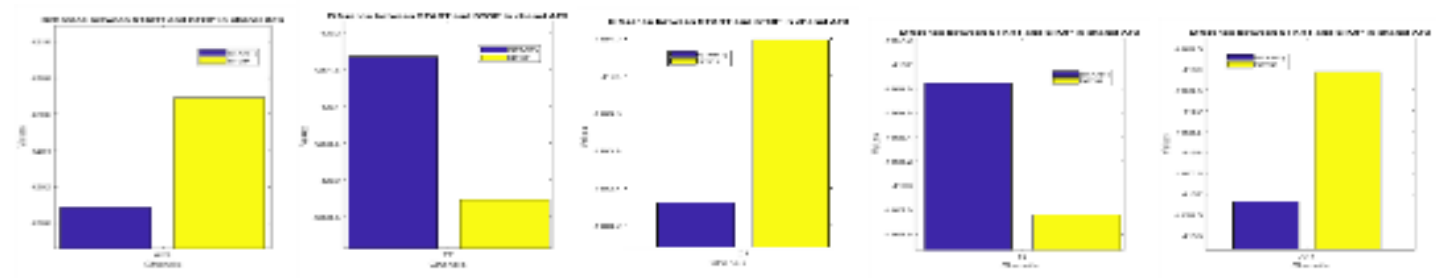

\section{Conclusions}

Identification of the separate brain signals and using this for interaction driven devices will increase in the next years but first steps are done.

In this research application of several data processing as filtering, calculation of average values, average deviation shows clear difference between channels of the signals for each command. This can be used for the creation of suitable algorithms for the processing in real time. Using similar methods and approaches this will give the opportunity, with the acquisition of an expansive database with suitable commands and their base characteristics for the commandeering of remote controlled devices.

Our team hopes in the future to build the necessary knowledge base for the various commands required for 
direct device management using brain signals

\section{Acknowledgements}

This work is partly supported by the project PPNIP-2018-15 "Creating an model of interface for the management of Smart Objects".

\section{References}

[1] Zhibin, T., William, H. B., \& Qianru, Z., (2013). Real-time EEG signal processing based on TI's TMS320C6713 DSK. Proceedings of the 120th ASEE Annual Conference@Exposition.

[2] Colombet, B., Woodman, M., Bénar, C. G., \& Badier, J. M. (2016). AnyWave: A cross-platform and modular software for visualizing and processing electrophysiological signals.

[3] Schalk, G., Brunner, P., Gerhardt, L. A., Bischof, H., \& Wolpawa, J. R. (2008). Brain-computer interfaces (BCIs): Detection instead of classification brain-computer interface research and development program. Journal of Neuroscience Methods, 167, 51-62

[4] Plesinger, F., Jurco, J., Halamek, J., \& Jurak, P. (2016). SignalPlant: An open signal processing software platform. Physiol Meas.

[5] Tara ThiagarajanIs the Emotiv EPOC signal quality good enough for research?, Retrieved December 1, 2016, from http://sapienlabs.co/emotiv-epoc-signal-quality-good-enough-research/

[6] Dennis, J. M., \& Jonathan, R. (2011). Wolpaw, brain-computer interfaces for communication and control. Communications of the ACM.

[7] Jaromir, S., Roman, Z., Roman, S., \& Roman. J. (2015). Using brain - Computer interface for control robot movement. Proceedings of the 29th European Conference on Modelling and Simulation.

[8] Murk, S., Rabel, T., Ahsan, Z., Muhammad, A. B., \& Hinesh, K. Wirelss Brain Computer Interface Streams Control Command.

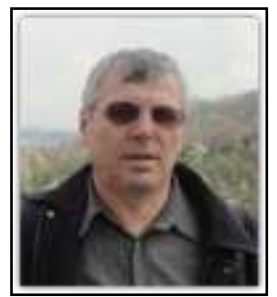

Georgi Petrov Dimitrov is a doctor of modeling and simulation process and a professor of computer science in the University of Library Studies and Information Technologies, Sofia, Bulgaria. Deputy Dean of the Faculty of Information Systems and Technologies. He currently works in area on Big Data, Web Data Analyst, IoT and etc. Prof. Dimitrov is the author of more 90 scientific publications, books and textbooks.

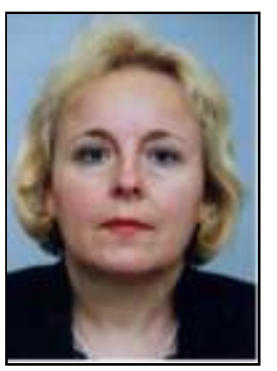

Galina Panayotova is a doctor of mathematics and professor of mathematical modeling in the State University of Library Studies and Informational technologies and University "Prof. Dr. As. Zlatarov"- Burgas.

Research interestsincluding: Nets and tissues in Finsler and Riemannian spaces; Application of differential geometry in the theory of PDE;Use of information technologies and applications in education; Mathematical and Computer Modeling. Prof. Panayotova is the author of more 80 scientific publications, books and textbooks.

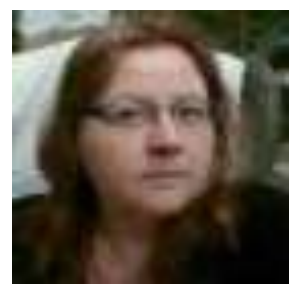

Eugenia Kovatcheva is an assoc. prof. at the University of Library Study and Information Technologies. She is a Scientific Secretary of the Dept. of Information Science. Her background is in the applications of Information Technologies and Cloud Computing in education, and training; adaptive learning systems. Her areas of interest are in UX design and Internet of Things, Digital Repository, Information, Knowledge and Innovation Management.

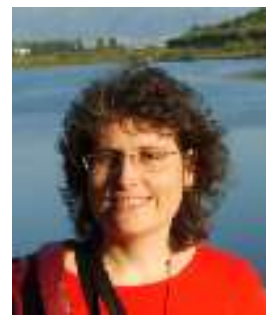

Daniela Borissova is currently associate professor at the Institute of Information and Communication Technologies of Bulgarian Academy of Sciences. She has specializations in Canada, Norway, Albania and Czech Republic. Her main research interests are related to 
decision support systems, modeling and optimization, e-learning, web design and development of web-based applications.

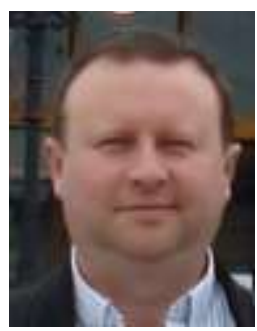

Pavel Stoyanov Petrov is a doctor of economics and associate professor of computer science in the "University of Economics - Varna", Varna, Bulgaria. Main research areas are: web security technologies, AI, business intelligence and etc. Assoc. Prof. Petrov is author of more than 60 scientific publications, books and textbooks. 